\title{
Talus reconstruction using fresh structural allograft after nontraumatic avascular necrosis: case report
}

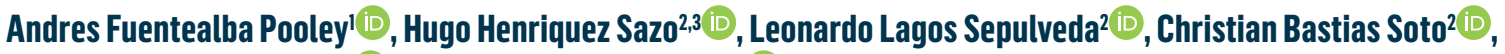 \\ Fernando Vargas Gallardo ${ }^{2} \mathbb{D}$, Sergio Fernandez Comber ${ }^{2} \mathbb{D}$ \\ 1. Orthopedics and Traumatology Service, Hospital de Urgencia y Asistencia Publica, Santiago, Chile. \\ 2. Orthopedics and Traumatology Service, Clínica Santa María, Santiago, Chile. \\ 3. Traumatology Institute Santiago - Universidad de Chile, Santiago, Chile.
}

\begin{abstract}
Avascular necrosis of the talus is a rare condition that can lead to important functional sequelae. There are few therapeutic alternatives for more advanced stages of this disease, the majority of which sacrifice the ankle joint. We report the case of a 50-year-old patient with nontraumatic avascular necrosis that compromised a large part of the talar surface. After non-structural autograft failed, it was reconstructed using fresh structural talar allograft. At one year of follow-up, the patient reported a considerable decrease in pain. To our knowledge, this is the first reported case in which fresh structural allograft was used in the treatment of nontraumatic avascular necrosis of the talus.
\end{abstract}

Level of Evidence V; Therapeutic Studies; Expert Opinion.

Keywords: Osteonecrosis; Talus; Allografts; Reconstructive surgical procedures.

\section{Introduction}

Avascular necrosis (AVN) of the talus is an interruption of irrigation that leads to necrotic areas of variable extent. It is a rare entity and its prevalence is unknown, since many patients only seek treatment in its late stages ${ }^{(1)}$.

Most often, vascular damage is secondary to a traumatic cause. However, there are cases of AVN without a history of trauma, for which multiple causes have been reported, including drugs or systemic pathologies. These nontraumatic causes represent $25 \%$ of all reported AVN cases $^{(2)}$.

There are multiple management alternatives in the treatment of AVN. Treatment will depend on the disease stage, as well as the severity and extent of the injury.

Fresh structural talar allograft has been traditionally used to manage osteochondral lesions (OCL) of the talus ${ }^{(3)}$. To our knowledge, this alternative has not been used in cases of massive nontraumatic AVN, probably due to the difficulty of the procedure and the lack of evidence on the subject. However, by preserving the joint, we believe that it would allow a greater level of functionality, which could be very important, especially in younger patients.

\section{Case Description}

This study was approved by the local institutional ethics committee. The patient granted consent to publish the data concerning her case.

We report the case of a 50-year-old female who presented with severe left ankle pain in August 2015. She had an iatrogenic lesion of the common right iliac artery during a spine surgery in 2010 that required emergency repair and a prolonged stay in an intensive care unit. The required vasoactive drugs led to multiple complications, including digital necrosis.

Five years after these events, she came to us complaining of anterior ankle pain, which increased during gait and ankle dorsiflexion. Subsequent imaging showed an important area of AVN in the left talus (Figure 1). 
The talus was reconstructed using non-structural autograft harvested from the ipsilateral iliac crest, with good results for pain and the patient's return to normal activities. However, 3 years after this surgery the patient returned, reporting anterior ankle pain that increased with gait. The physical examination showed important swelling on the left ankle, associated with pain during maximum ankle dorsiflexion or plantar flexion.

Computed tomography revealed a massive AVN of the left talus associated with graft resorption and a significant depression in the lateral two-thirds of the talus (Figure 2). It was then decided to resect the left hemitalus and transplant fresh structural talar allograft according to the surgical technique described below.

\section{Surgical technique}

The anterior ankle approach was used. A large osteophyte on the anterior tibia was resected, allowing visualization of a large area of damaged cartilage on the articular surface of the talus, including an important depression in the lateral weight-bearing surface.
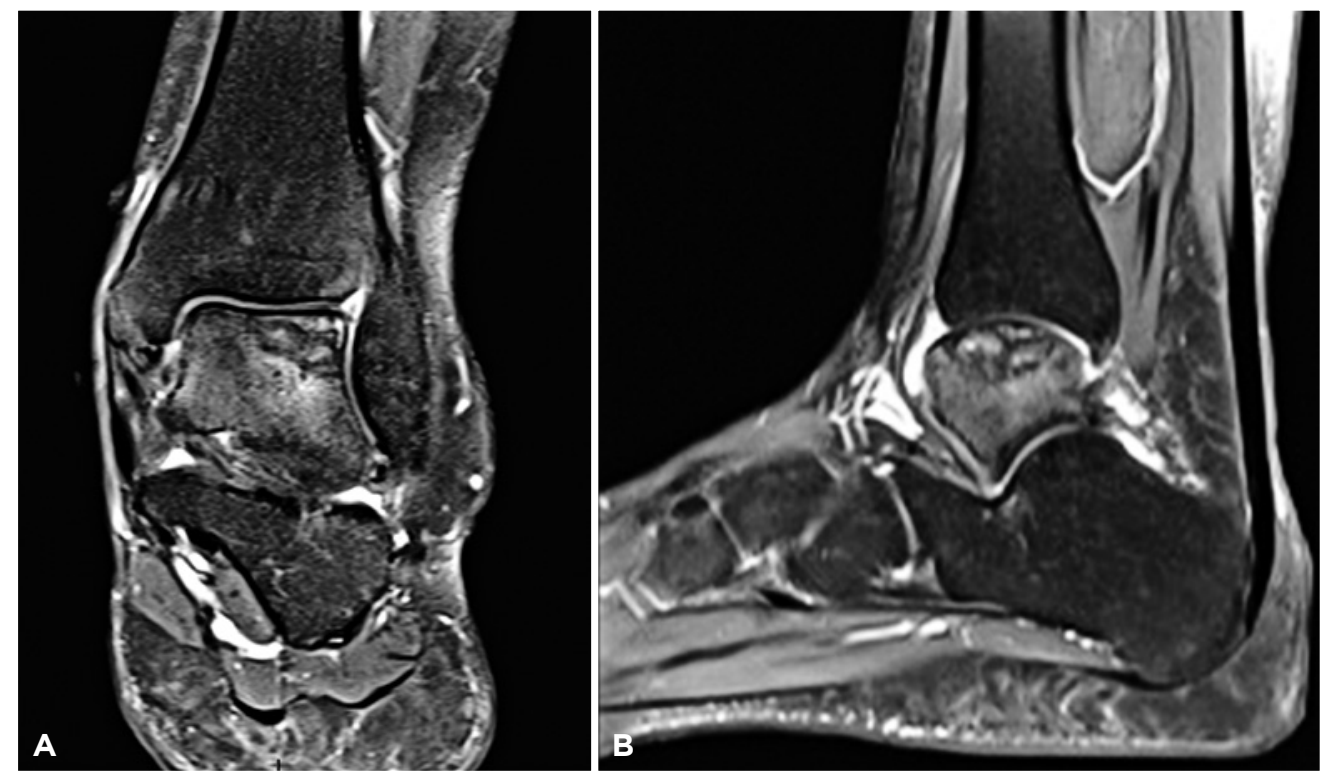

Figure 1. MRI of the ankle at initial patient presentation. Coronal (A) and sagittal (B) sections show the avascular necrosis of the talus, which affects approximately half of its articular surface.
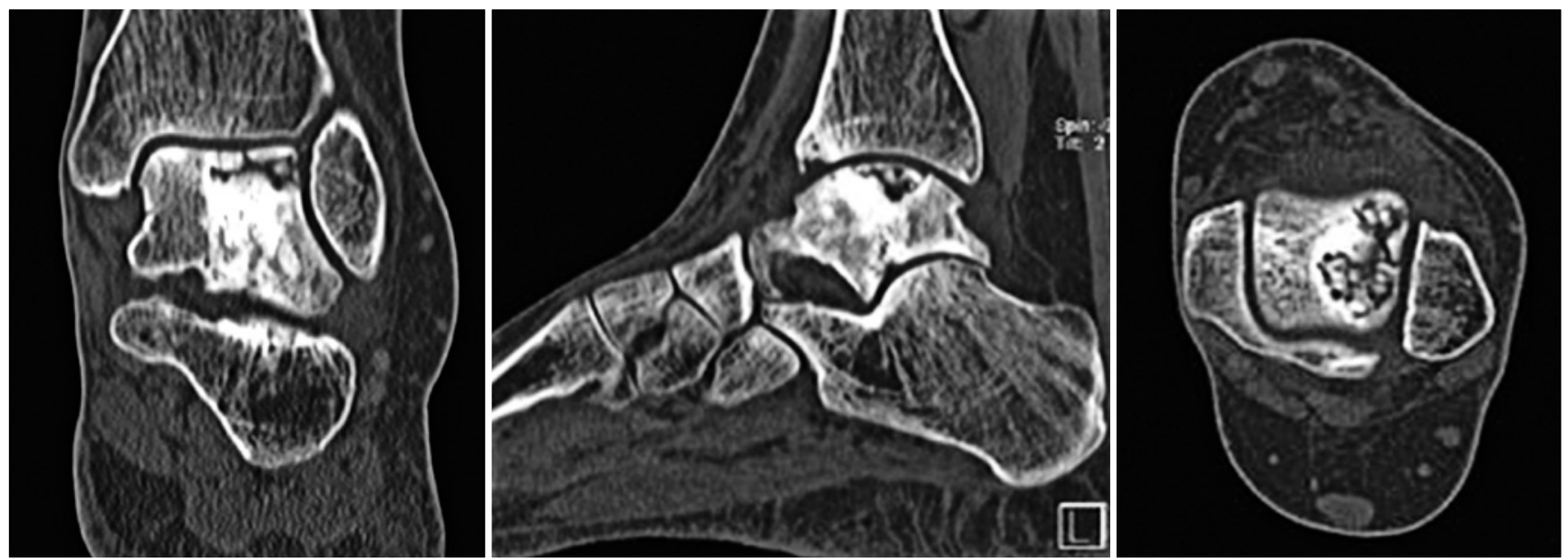

Figure 2. Computed tomography of the ankle three years after reconstruction with iliac crest autograft, showing massive avascular necrosis of the lateral two-thirds of the talus, associated with graft resorption. 
An AO distractor was applied to improve the exposure of the compromised area and an osteotomy of two-thirds of the lateral articular surface of the talus was performed, with radioscopy-guided incisions.

The recipient area was precisely measured and the talar allograft was adjusted with a bone saw to match the defect. The allograft's fit in the recipient site was then confirmed, achieving good joint reduction (Figure 3 ).

The allograft was fixed with 3 Zimmer Biomet ${ }^{\oplus}$ bioabsorbable screws $(2.5 \mathrm{~mm})$; the screw heads were removed to avoid prominences in the joint area.

The wound was closed with absorbable sutures for subcutaneous tissue and monofilament for the skin. The ankle was covered with sterile gauze and a bandage and immobilized with a controlled ankle motion boot.

One year after surgery, the patient reported a $50 \%$ reduction in pain compared to her preoperative state, although she had not completely returned to work activities due to an ina- bility to remain standing or walking for long periods. At this point, her Foot and Ankle Ability Measure was 54\% and her American Orthopedic Foot \& Ankle Society Ankle (AOFAS) Hindfoot Scale score was 65. Computed tomography showed partial consolidation of the allograft (Figure 4).

\section{Discussion}

Managing AVN of the talus is complex ${ }^{(1,2)}$. The pathogenesis and etiologies of this disease have been better understood in recent years, increasing the therapeutic options. However, the results are still quite poor in terms of clinical outcomes ${ }^{(2)}$, with arthrodesis performed as a definitive solution in many cases $^{(4,5)}$.

When faced with this pathology, a fuller understanding of the natural evolution of the disease is required. The most commonly used staging system is that of Ficat and Arlet ${ }^{(6)}$, which has been modified over the years and currently includes: a preclinical stage (stage 0), a preradiographic stage
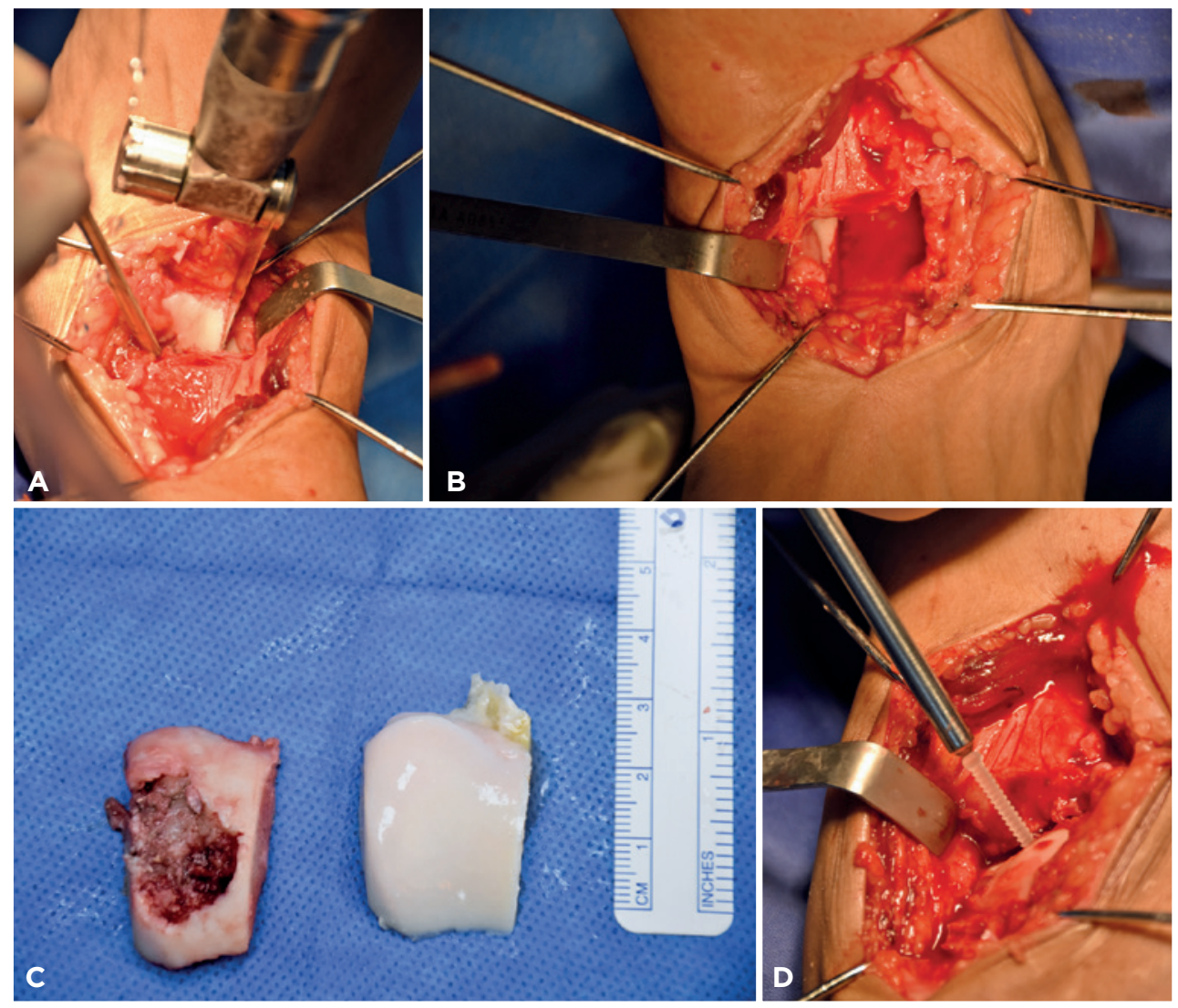

Figure 3. Intraoperative photographs of the procedure. A) Previously measured cuts of the native talus were performed with a bone saw; B) The recipient site after resecting the necrotic talus, corresponding to the lateral two-thirds of the articular surface; C) Comparison of the resected native talus with the fresh talar allograft. D) Allograft fixation with Zimmer - Biomet ${ }^{\circledast} 2.5 \mathrm{~mm}$ bioabsorbable screws. 

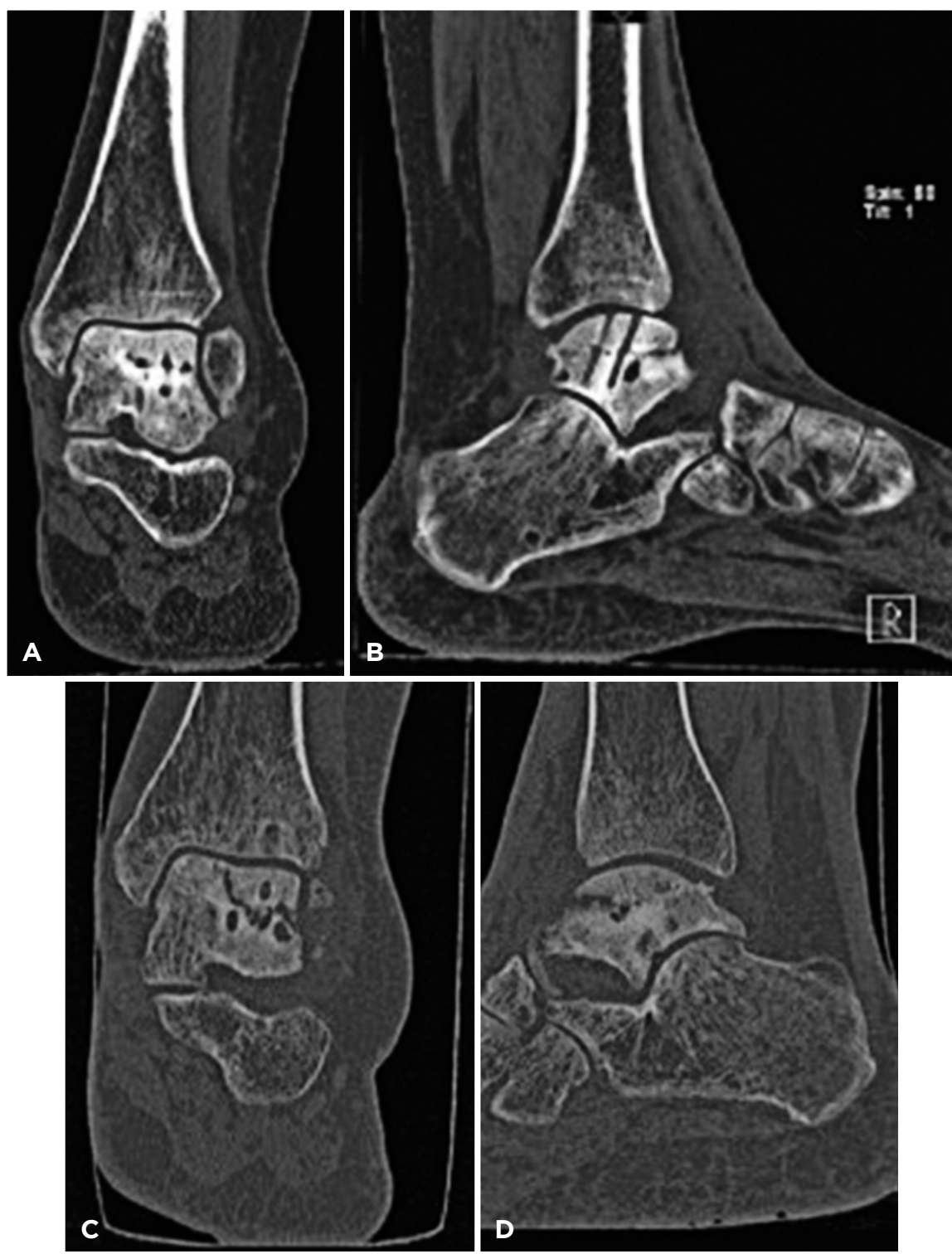

Figure 4. A and B: Computed tomography scans at first postoperative control: the allograft is in a good position, congruent with the articular surface. C and D: Computed tomography scans 1 year postoperatively: the allograft is partially integrated, with some graft resorption. The joint surface is regular, with no collapse of the allograft.

(stage I), pre-collapse with radiological changes (stage II), a transition phase with flattening of the articular surface and collapse with intact surrounding joints (stage III), and finally osteoarthritis (stage IV)(1). The therapeutic options are determined by the stage of the disease ${ }^{(1,5)}$. In advanced stages, specifically when the talus has collapsed, more radical options that usually sacrifice the joint are recommended, such as ankle arthrodesis. Joint-sparing procedures, such as ankle arthroplasty, do not show predictable results in advanced AVN of the talus ${ }^{(1,4,5)}$.
Gross et al. ${ }^{(5)}$ conducted a systematic review to compare therapeutic options for AVN of the talus, concluding that conservative management should be attempted initially with restricted weight-bearing either with or without extracorporeal shock wave therapy. However, when nonoperative management fails, they recommend surgical management. For patients in Ficat and Arlet stages I-III, they recommend core decompression or non-structural autograft, reserving arthrodesis for the final stages of the disease or when previous treatments have failed ${ }^{(5)}$. 
Tibiotalar arthrodesis is a good option for advanced stages and has good results in terms of functional scales ${ }^{(4)}$. However, its disadvantage is lost joint mobility, limiting the patient's gait to different degrees. It is also not exempt from complications: depending on the case series, non-union rates can from $18 \%-40 \%{ }^{(5)}$.

Studies describing the use of fresh structural talar allograft are mainly conducted in patients diagnosed with OCL of the talus. It is normally indicated for large lesions, ie, more than $1.5 \mathrm{~cm}$ in diameter or an area greater than $150 \mathrm{~mm}^{(3)}$.

Adams et al. ${ }^{(7)}$ conducted a prospective study of 14 patients with OCL of the talus who were managed with fresh structural allograft, following them for 2 years. They reported an overall success rate of $86 \%$, with significant improvement in terms of pain reduction (visual analog scale) and functional scales, obtaining a mean postoperative AOFAS score of 84 points. However, 5 patients (36\%) in this series required some additional surgery to manage pain or stiffness ${ }^{(7)}$.

Raikin ${ }^{(8)}$ conducted another prospective study on 15 patients with cystic OCL of the talus who were managed with fresh structural allograft. The mean volume of these lesions was $6059 \mathrm{~mm}^{3}$, and the mean AOFAS ankle-hindfoot score was 83 points. Only two patients required ankle arthrodesis due to allograft failure.

Comparing our case with the literature, the results after 1 year of follow-up are acceptable, although the graft had not completely consolidated and the results of functional scales were moderate.

The delay in graft integration may be due to the pathophysiology of AVN, in which, unlike traumatic OCL, the complex vascular network that irrigates the talus may be affected. Thus, there would be a decrease in vascular supply to the grafted area, which could affect its osseointegration.
Using vascularized bone grafts may prevent this problem. Yu et al. ${ }^{(9)}$ reported good results with vascularized cuneiform bone flap plus iliac cancellous bone grafting in Ficat and Arlet stage II, III, and IV patients with non-traumatic AVN of the talus. Specifically, the results were excellent in $90 \%$ of cases. However, these are technically very complex procedures, and their usefulness is mainly in small lesions up to Ficat and Arlet stage III (10)

Over a mean follow up of 6 years, Nunley et al.(11) reported that rotational vascularized pedicle bone graft from the cuboid had good results in terms of pain relief and physical function in $84 \%$ of their Ficat and Arlet stages II and III patients.

The delay in osseointegration in the present case could also be explained by the size of the graft, since the necrosis affected two-thirds of the lateral talus, with a volume of $8932 \mathrm{~mm}^{3}$ according to computed tomography. This is considerably higher than other reports ${ }^{(7,8)}$.

However, although radiological consolidation of the graft had not occurred by 1 year of follow-up, our patient reported significant improvement compared to her preoperative state.

This study is limited by its retrospective design and by including a single case. The follow-up time should also be considered a limitation.

\section{Conclusion}

Although there is scant literature on this condition, we obtained favorable results with this technique in a patient with AVN of the talus. Further research with a higher level of evidence could confirm this technique as a management alternative, especially for young patients with massive lesions, for whom arthrodesis can be prevented or delayed. 


\section{References}

1. Haskell A. Natural History of Avascular Necrosis in the Talus: When to Operate. Foot Ankle Clin. 2019;24(1):35-45.

2. Adelaar RS, Madrian JR. Avascular necrosis of the talus. Orthop Clin North Am. 2004;35(3):383-95.

3. Gross CE, Adams SB, Easley ME, Nunley JA. Role of Fresh Osteochondral Allografts for Large Talar Osteochondral Lesions. Instr Course Lect. 2016;65:301-9.

4. Lachman JR, Adams SB. Tibiotalocalcaneal Arthrodesis for Severe Talar Avascular Necrosis. Foot Ankle Clin. 2019;24(1):143-61.

5. Gross CE, Haughom B, Chahal J, Holmes GB. Treatments for Avascular Necrosis of the Talus: A Systematic Review. Foot Ankle Spec. 2014;7(5):387-97.

6. Ficat RP, Arlet J. Forage-biopsie de la tete femorale dans l'osteonecrose primative. Observations histo-pathologiques portant sur huit forages. Rev Rhum. 1964;31:257-64.

7. Adams SB, Dekker TJ, Schiff AP, Gross CP, Nunley JA, Easley ME.
Prospective Evaluation of Structural Allograft Transplantation for Osteochondral Lesions of the Talar Shoulder. Foot Ankle Int. 2018;39(1):28-34.

8. Raikin SM. Fresh osteochondral allografts for large-volume cystic osteochondral defects of the talus. J Bone Joint Surg Am. 2009;91(12):2818-26.

9. Yu X, Zhao D, Sun Q, Wang T, Yu H, Su Y, et al. [Treatment of nontraumatic avascular talar necrosis by transposition of vascularized cuneiform bone flap plus iliac cancellous bone grafting]. Zhonghua Yi Xue Za Zhi. 2010;90(15):1035-8.

10. Dhillon MS, Rana B, Panda I, Patel S, Kumar P. Management Options in Avascular Necrosis of Talus. Indian J Orthop. 2018;52(3): 284-96.

11. Nunley JA, Hamid KS. Vascularized Pedicle Bone-Grafting from the Cuboid for Talar Osteonecrosis: Results of a Novel Salvage Procedure. J Bone Joint Surg Am. 2017;99(10):848-54. 